\title{
Bullshit jobs: Graeber y la alienación en el capitalismo financiarizado
}

\author{
Bullshit Jobs: Graeber on Alienation \\ within Financialised Capitalism
}

ÁLVARO RAMOS COLÁS (Universidad Nacional de Educación a Distancia)

Artículo recibido: 26 de junio de 2018

Solicitud de revisión: 26 de noviembre de 2018

Artículo aceptado: 15 de diciembre de 2018

Ramos Colás, Álvaro (2019). Bullshit jobs: Graeber y la alienación en el capitalismo financiarizado. Revista de Pensament i Análisi, 24(1), pp. 134-154.

\section{Resumen}

En el presente ensayo intentaremos exponer algunos aspectos de la crítica de Graeber al capitalismo financiarizado. En el primer apartado introduciremos su concepto de alienación, clave para entender el sentido de su obra. En los apartados posteriores presentaremos las ideas principales que se pueden encontrar en Bullshit Jobs, su último libro. En él expone una teoría de los trabajos basura o inútiles, que considera uno de los pilares fundamentales de la ideología neoliberal, junto con la omnipresencia de la burocracia y la moralidad de la deuda. El fenómeno social de los trabajos basura es estudiado desde diversos niveles: qué son, cómo afectan a los que los realizan, cuáles son las causas económicas y por qué nadie hace nada para acabar con ellos.

Palabras clave: trabajo basura, anarquismo, revolución, neoliberalismo, alienación.

\section{Abstract}

In the present paper I try to delve into some of Graeber's critiques of the financialised capitalism. In the first section, I will introduce his idea of alienation, key to understand the sense of his work. In the rest of the paper I will present the main ideas of his last book, Bullshit Jobs. There Graeber presents a theory of bullshit jobs, which he believes to be an important base of the neoliberal system, along with the bureaucracy and the debt. He analyses the social phenomenon of bullshit jobs from different perspectives: what they are, how they affect the people doing them, which are the economic causes and why nothing is done. 
Key Words: bullshit job, anarchism, revolution, neoliberalism, alienation.

\section{INTRODUCCIÓN}

Una de las consecuencias de la visión abstracta de la realidad social que nos presenta le teoría económica, tal y como el profesor Palazuelos nos muestra (Palazuelos, 2015: 17), es la separación de lo económico y lo político. Por un lado, tenemos el reino de la economía, definido por las empresas, la riqueza, los mercados y la eficiencia; por otro, el de la política, determinado por los Estados, el poder, las instituciones y la estabilidad. Son órdenes distintos, pero hay una diferencia de rango, a saber: la política debe garantizar que la economía funcione, esa es su misión.

Gran parte de la producción intelectual de Graeber está dirigida a desmentir la supuesta inmutabilidad de la teoría económica más abstracta y matematizada. En Deuda, por ejemplo, demostró que, cuando hablamos de deuda en el mundo capitalista actual, no solo lo hacemos en términos actuarios y contables, sino también y especialmente en términos morales. Del mismo modo, en La utopía de las normas, trajo a colación cómo mediante la burocracia el sistema sofoca cualquier posibilidad de pensamiento alternativo. En su reciente trabajo, Bullshit Jobs, el antropólogo americano abunda en la moral del trabajo, a la que considera otro de los cimientos de la ideología neoliberal.

En consecuencia, podemos entender la obra de Graeber como un intento de rebatir la supuesta división entre economía y política a través del estudio de los mecanismos que operan en ella. En el presente ensayo intentaremos profundizar en este aspecto de su obra y, más en concreto, en el desenmascaramiento de uno de los tópicos de la ideología neoliberal, la moral del trabajo. Graeber defiende que, detrás de todos los axiomas económicos que sustentan el capitalismo actual (obligatoriedad de pagar lo que se debe - la deuda-, la omnipresencia de la burocracia y la dependencia psicológica del trabajo), se encuentra una posición ético-política y no estrictamente económica, tal y como la teoría económica sostiene. La apariencia de ciencia con la que se ha investido a la economía esconde, a su juicio, las verdaderas motivaciones de las élites como son la apropiación del excedente y el control sin fisuras de la esfera social. Su obra trata a grandes rasgos de sacar a la luz, desde la antropología, estas contradicciones. 


\section{ALIENACIÓN Y BUROCRACIA}

El antropólogo norteamericano centra su ámbito de investigación en las formas concretas en las que el capitalismo del siglo XXI somete a los individuos, y que se lleva a cabo mayormente mediante la burocracia, la deuda y el trabajo. Esto nos lleva directamente al concepto de alienación, el cual es tratado en mayor profundidad en su libro sobre la burocracia, La utopía de las normas. En este apartado veremos qué entiende él por alienación a partir de dicho texto para, de este modo, poder adentrarnos un poco más en detalle en el papel, también decisivo, que juega el trabajo para Graeber en el mantenimiento y la reproducción del sistema capitalista moderno.

En La utopía de las normas ${ }^{1}$ profundiza en cómo la burocracia mediatiza cada aspecto de nuestra vida diaria (Graeber, 2013a: 289) sometiendo la imaginación, que es lo más característico de nuestra naturaleza humana.

Si existe una esencia humana, es precisamente nuestra capacidad para imaginar que tenemos una esencia. En esto no me encuentro en absoluto alejado del punto de partida de Marx: lo esencialmente humano, es la capacidad para imaginar cosas y hacerlas realidad [...], y que la alienación ocurre cuando perdemos el control de ese proceso (Graeber, 2009: 525526. Cursivas nuestras).

Graeber se refiere al Marx de los Manuscritos..., para el que el trabajo no alienado es conceptualizado como una actividad eminentemente creativa que define a los hombres y a las mujeres. El ser humano es imaginativo, es capaz de analizar problemas, interpretar situaciones, buscar soluciones y llevarlas a cabo. Desde el momento en que se nos priva de estas capacidades, perdemos nuestra esencia humana. En definitiva, estamos alienados. Como veremos, en opinión de Graeber, este es el modo en que el capital nos domina y que toma especial relevancia en la burocracia, en la deuda y en el trabajo. Este dominio, según Graeber, sofoca la imaginación del ciudadano, y así perpetúa su dominio total, que es ejercido de manera piramidal, esto es, de arriba abajo y sin dejar (casi) ningún aspecto fuera su control. Así se genera lo que Graeber denomina «violencia estructural», puesto que crea estructuras asimétricas de la imaginación en las que una élite puede dedicarse a trabajos eminentemente creativos,

Agradezco a Ramón del Castillo esta referencia bibliográfica, así como otras muchas de las utilizadas en este artículo. 
mientras una gran masa de trabajadores se limita a trabajos rutinarios y, como veremos, improductivos (Graeber, 2015: 84).

La estructura social violenta se caracteriza, en primer lugar, porque los de abajo realizan la mayor parte del trabajo real y físico, pero también (y esta es una aportación importante de Graeber) porque estos empeñan gran parte de su tiempo en interpretar las estructuras sociales que sustenta su precariedad. La élite impone las reglas y se preocupa de que sean cumplidas mediante la coacción, quedándole a la masa trabajadora alienada solo la opción de rebelarse si aspira a una vida verdaderamente humana. Una vez implementado el sistema, podría decirse que es una maquinaria que funciona como un autómata, ya que el mero hecho de la dependencia material y psicológica y de la amenaza de la represión hace prácticamente imposible replantearse un modelo social alternativo. Por eso afirma Graeber que los individuos situados en la base de la pirámide emplean casi todas sus capacidades interpretativas y creativas en darle un sentido a toda esa maraña burocrática que regula sus vidas en vez de en intentar repensar todo el sistema por sí mismos, porque, al verse sometidos y sin posibilidad de escapatoria, intentan encontrar la lógica al régimen que, realmente, no tiene (más allá de la lógica de la dominación).

El resultado de la experiencia vital de la alienación, que es para Graeber el rasgo más característico del capitalismo actual, supone la pérdida de control de nuestros procesos vitales más elementales, a saber, la convivencia, el trabajo, la comunicación a todos los niveles, la resolución de conflictos, etc. Esa pérdida es lo que entiende nuestro autor por alienación, tal como hemos visto en la cita anterior. Podría entenderse también como la privación por la fuerza y para beneficio de las élites de nuestra esencia más humana: la imaginación y la creación. Richard Sennett (2000, 2006), por ejemplo, haciendo gala de una especial sensibilidad, ha sabido retratar esta sensación de opresión (a pesar de que el discurso neoliberal afirme defender la libertad sobre todas las cosas) de sentirse despojado de lo más personal y de la imposibilidad de llevar a cabo un proyecto vital pleno. Esto atenaza a los trabajadores de muy distinta extracción en el capitalismo posfordista y eso es, en gran medida, a lo que se refiere Graeber cuando habla de alienación. ${ }^{2}$

El pensamiento de izquierdas, para Graeber, parece haber dado la espalda a la cuestión de la alienación que conlleva la burocracia. Es cierto que las revueltas del 68 elevaron la imaginación al estatus de elemento revolucionario

Aunque también hay que señalar que Sennett, en ningún caso, extrae las consecuencias revolucionarias de Graeber. 
de primer nivel («la imaginación al poder»), pero Graeber subraya que, precisamente, fueron las generaciones que participaron en ellas (tan influenciadas por el pensamiento inmediatamente posterior al 68) las que dieron la espalda a la imaginación como forma de vida y, por tanto, impidieron el fin de la alienación en el sentido que él la entiende. En concreto, Graeber carga las tintas contra el posestructuralismo francés de Foucault, Deleuze o Baudrillard, el cual fue adoptado por la academia (ironías del destino) como discurso hegemónico, acallando voces como las de los situacionistas, más críticas y beligerantes con el sistema (Graeber, 2009: 259). El pensamiento posterior al 68 es caracterizado por Graeber como un discurso descriptivo, recluido en los departamentos universitarios y en manos de mandarines encargados de proteger su tradición, mientras que el de Bookchin, Debord y, sobre todo, Vaneigem representa un canto a la acción y al cambio radical en todos los aspectos de nuestras vidas cotidianas. En concreto, el texto de mayor influencia en Graeber es La revolución de la vida cotidiana (1967) de Vaneigem, donde el revolucionario belga despliega sus puntos de vista sobre la alienación y la revolución, que a la postre tendrán una influencia decisiva en el antropólogo norteamericano y su modo de entender la alienación como una experiencia subjetiva de poder y dominio:

«alienación» es lo que se siente cuando nuestra concepción de valor (lo que creemos que debemos esperar de la vida, lo que consideramos importante o apreciable, etc.) choca radicalmente con los estándares dominantes (Graeber, 2009: 280).

En definitiva, lo que Graeber propone no es una lucha por una mejora de la sociedad, sino por una sociedad donde los distintos modos de valorar sean tenidos en cuenta. No niega con esto que no haya situaciones en el presente que puedan y deban ser rectificadas (reducciones de las jornadas laborales, mejoras en las coberturas sociales, etc.), sino que el único modo de hacer una verdadera revolución es la lucha contra la alienación.

La burocracia y su fuerza coactiva es, a juicio de Graeber, una de las maneras más eficaces de imponernos estos valores, puesto que cala hasta en el aspecto más íntimo de nuestra vida privada. Por eso considera que la revolución debe cuestionar todos sus aspectos, pero no de manera individual, sino en su conjunto, es decir, debería ser una enmienda a la totalidad, a la manera que imaginamos y valoramos nuestras vidas en común. 


\section{DEFINICIÓN Y EFECTOS PERSONALES DEL TRABAJO BASURA}

¿Qué es un trabajo basura?' Lo primero que se nos viene a la mente es un trabajo en condiciones precarias, mal pagado, a tiempo parcial y determinado, que pone en riesgo la integridad física del trabajador, etc. En lo que llevamos de década, Guy Standing (2013, 2017a) ha arrojado luz sobre esta realidad y sus consecuencias, gracias a su conceptualización del precariado como nueva clase oprimida de un modo distinto por verse condenada a este tipo de empleos. Si bien Standing caracteriza al precariado como clase social en El precariado. Una nueva clase social, su posición ha sido en ocasiones ambigua. Es cierto que la categoría del precariado es heterogénea, pero él defiende que también lo es el proletariado. Los elementos que, según él (2017b), definen al precariado como clase son: a) su relación distintiva con la producción: todos sus miembros aceptan de algún modo la inseguridad en el trabajo; $b$ ) consagran gran parte de su tiempo a la búsqueda de trabajo, tarea que, lógicamente, no es remunerada; c) no tienen un relato para explicarse su vida. De esta manera, la explotación del precariado, a diferencia de la del proletariado, no es primariamente laboral, sino que se extiende más allá de lo económico y lo material. En el fondo, Graeber defiende algo parecido con las nociones de alienación y violencia espiritual, es decir, el capitalismo financiarizado tiene más y más sofisticadas armas para controlar a los que explota.

De todos modos, Graeber procura ir más allá de la crítica cualitativa y de sus consecuencias; lo que tiene en mente, como esperamos poder mostrar a lo largo de este ensayo, es realizar una enmienda a la totalidad de la realidad capitalista, pero sobre una base moral que, por su puesto, tiene consecuencias materiales. Podría decirse que autores como, por ejemplo, Standing en sus tesis sobre el precariado (2013) y Srnicek y Williams (2015: 123-154) ${ }^{4}$ parten de

3 La traducción de bullshit job es un tanto compleja. Traducirlo como 'trabajo superfluo' capta la futilidad de este tipo de trabajos a la que se refiere Graeber, pero no el toque despectivo y de lenguaje llano del adjetivo inglés. Justo por cargar demasiado las tintas en este último aspecto, traducirlo como 'trabajo de mierda', 'gilipollesco' o incluso 'pamplinas' si se quiere no suena demasiado bien al lector castellanoparlante. Por estos motivos proponemos un camino intermedio, como el de 'trabajo basura', a pesar de ser conscientes de que no capta por completo el sentido de bullshit.

4 Srnicek y Williams realizan un análisis de la precariedad desde una perspectiva de un futuro anticipado, esto es, la situación actual de precariedad anticipa los peores escenarios venideros, por eso afirman que «el futuro no está funcionando» (2017: 123) y de ahí la necesidad de inventar el futuro. En concreto, presentan la figura del paupervirtual, que o bien es explotado en su puesto de trabajo (con unas condiciones laborables peores que las que, en general, se tenían en el fordismo) o ni tan siquiera son explotados y, en consecuencia, su situación es todavía más precaria desde una perspectiva material. 
lo material (la mala calidad del trabajo y las limitaciones reales que sufre el trabajador en temas de salud, salario, servicios de primera necesidad, etc.) para llegar a lo moral (estas limitaciones son injustas), mientras que Graeber, a la inversa, parte de la crítica moral para llegar a presentar algunas de las consecuencias materiales.

Segunda definición provisional: un trabajo basura es una forma de empleo que es tan absolutamente inútil, innecesario o pernicioso que incluso el mismo empleado no puede justificar su utilidad y su existencia, aunque éste se sienta obligado a fingir que no es su caso (Graeber, 2018: 8).

Pero esta definición es, tal como indica el mismo autor, provisional. Hay otro aspecto clave que la completa y que, además, es el que nos va a acercar al carácter moral de la crítica de Graeber: «mi definición es principalmente subjetiva. Un "trabajo basura" debe ser percibido como inútil, innecesario o pernicioso por el trabajador» (Graeber, 2018: 10, cursivas nuestras). Esta percepción del trabajador es clave para entender la diferencia a la que aludíamos anteriormente entre una basura de trabajo y un trabajo basura. Los primeros son, en efecto, los precarios y que afectan a las capas más débiles del mercado laboral: jóvenes, desempleados mayores de 50 años, madres solteras, etc. Los trabajos basura a los que se refiere Graeber afectan principalmente al personal de cuello blanco, a personas que pueden reflexionar acerca del sentido de lo que hacen sin estar mediatizados por la necesidad material que les cubre el buen sueldo de su empleo. Conviene insistir en que Graeber no niega ni la importancia de trabajos precarios ni sus deleznables consecuencias (aunque también es cierto que no los analiza en este libro), sino que defiende que, normalmente, en ese tipo de actividades se aporta un verdadero valor, es decir, son útiles a pesar de estar mal retribuidos.

Hay una gran variedad de trabajos basura, especialmente entre los cargos intermedios del sector terciario. Graeber dedica un capítulo entero a mostrarnos los grandes grupos en los que los podemos agrupar como, por ejemplo, los lacayos (flunkies), cuyo objetivo es hacer que alguien que ocupe un puesto relativamente importante en la pirámide social se sienta importante (consejeros, organizadores de agendas, secretarios, etc.); otro ejemplo sería el de los matones (goons), que tienen un papel que implica violencia o amenaza de violencia pero que son empleados aunque solo sea por su capacidad intimidatoria (lobistas, abogados corporativos, etc.). 
¿Por qué debería afectarles la inutilidad de sus empleos? Porque, en opinión de Graeber, este tipo de empleos tiene un impacto moral y espiritual devastador en la mayoría de los que los realizan. Por un lado, el trabajador pierde su esencia humana creadora e imaginativa que, para nuestro autor (siguiendo al joven Marx), conforma al ser humano. Este grupo de personas, más amplio de lo que parece, 5 sufre, a la luz del concepto de alienación que hemos presentado más arriba, lo que el autor llama «violencia espiritual». Es violencia puesto que estas ocupaciones privan a ciertos individuos de su propia naturaleza humana al alienarlos, y se puede adjetivar como espiritual por su efecto sobre la moral, la salud psicológica, la motivación, el desarrollo personal, etc.

En toda la obra de Graeber late el impulso de recuperar, precisamente, la dignidad humana, su esencia, frente a ciertas instituciones que, aunque parecen inamovibles y casi naturales («esto es así porque no puede ser de otra manera»), no son sino convenciones que favorecen a una élite y que, directa o indirectamente, les otorga beneficios económicos y privilegios sociales. Se podría decir, por tanto, que su obra es una lucha contra la alienación y la violencia espiritual que ejerce.

En Bullshit Jobs, el antropólogo norteamericano profundiza en el impacto personal de los trabajos basura, en las experiencias traumáticas que conllevan y que las personas no saben cómo asimilar. Lógicamente, al haber gran variedad de trabajos de este tipo, las maneras en que afectan a los trabajadores son muy variadas (Graeber, 2018: 113 y ss.), ${ }^{6}$ por ejemplo, suele ser muy difícil de asimilar el hecho de no ser la causa de nada; también es duro aceptar que mi actividad suponga una rémora para la empresa (y, en consecuencia, para la sociedad) o sentirse indigno de la desdicha que este tipo de trabajos lleva a menudo aparejada, máxime teniendo un buen sueldo y en cierto modo reco-

5 Graeber aporta algunos datos, a nuestro juicio insuficientes, en Bullshit Jobs. El primero es una estadística realizada por YouGov, de la que no ofrece ninguna referencia exacta, en la que se le preguntaba en el Reino Unido a los entrevistados si creían que su trabajo «realizaba una contribución socialmente relevante». El 37 \% respondió que no. Otra parecida se llevó a cabo en Holanda, la cual arrojó un 40 \% de respuestas negativas. Esto, para Graeber, es «una prueba estadística incontestable» de la importancia de su tesis (2018: xxi-xxii). Más adelante (2018: 146 y ss.), presenta cuatro gráficas que muestran cómo el sector servicios se ha convertido en los últimos cien años en el sector abrumadoramente predominante. Teniendo en cuenta que la mayoría de los trabajos basura se realiza en el sector terciario, este hecho le sirve como argumento para reforzar su idea de la importancia de este tipo de trabajos en las sociedades actuales. En cualquier caso, el aparato estadístico parece demasiado escaso para poner en perspectiva la relevancia del hecho estudiado.

6 La mayoría de los casos de los que extrae sus ideas son de terceras personas que le han contactado directamente después de la publicación del artículo «On the Phenomenon of the Bullshit Jobs: A Work Rant». https://strikemag.org/bullshit-jobs/ 
nocimiento social (poca gente sospecha que tu trabajo es inservible, simplemente no saben cuáles son tus tareas en el puesto de trabajo).

Estas son algunas de las consecuencias de la violencia espiritual que ejerce la alienación de los trabajos basura y que nos ayudan a entenderla. Podría decirse, en resumen, que la violencia espiritual es la privación por la fuerza de la creatividad, que es la capacidad que nos define como humanos según Graeber. En los siguientes apartados veremos los motivos económicos y culturales que el autor aduce para que surjan, y las razones culturales para que no se luche contra ellos.

La alienación que nos presenta Graeber es novedosa porque contrasta con la que han estudiado importantes sociólogos actuales en una dirección, en cierto modo, contraria. Por ejemplo, Francis Green estudia la pérdida de calidad del trabajo, que toma forma en la intensificación en el posfordismo actual (Green 2004, 2007; Green, Felstead y Gallie, 2015). Esta mayor intensidad conlleva una mayor exigencia, el trabajador es obligado a realizar más tareas en las mismas horas de jornada laboral por un sueldo que no crece tanto como las responsabilidades. Esto redunda en un menor impacto del empleado en la empresa, una mayor flexibilización en los cometidos diarios y una desigualdad en la calidad de los trabajos (Green, Felstead y Gallie, 2015). La paradoja que se da en grandes estratos del mercado laboral del primer mundo es que, a pesar de exigir a los trabajadores cada vez mayores capacidades, estos se ven relegados a actividades más numerosas, que implican una mayor ocupación de su tiempo pero que imposibilitan el despliegue de esas capacidades.

Cristophe Dejours (2015), desde una perspectiva algo diferente, ha profundizado en las consecuencias psicológicas de la falta de confianza y de reconocimiento que sufren los empleados. Es lo que él denomina como «psicodinámica del reconocimiento", en la que entran en juego la subjetividad del asalariado, la acción de otros sujetos que también anhelan el reconocimiento y las regulaciones genéricas de cualquier estructura laboral.

En cambio, la paradoja que nos presenta Graeber afirma que los trabajadores no se sienten útiles porque su trabajo es superfluo, es decir, porque realmente no hacen nada productivo, lo cual los aliena al coartar lo más humano que hay en ellos: la capacidad de trabajar e imaginar. Las posiciones de Green, Dejours y Graeber pueden parecer en algunos aspectos opuestas, pero también podrían interpretarse como complementarias. Teniendo en cuenta la naturaleza proteica del neoliberalismo, es muy probable que estos fenómenos (trabajos cada vez más extenuantes y menos humanos y trabajos basura) convivan en un mismo país, en un sector e incluso en una misma empresa. Poner 
estas tesis en perspectiva nos aporta un nuevo ángulo desde el que entender cómo el neoliberalismo contemporáneo aliena de modos muy diversos y complementarios a los trabajadores.

\section{TRABAJO BASURA Y SU EXPLICACIÓN ECONÓMICA}

Para situar algo más en perspectiva la teoría de Graeber acerca del trabajo, es interesante compararla con la del influyente El nuevo espíritu del capitalismo de Boltanski y Chiapello (2002), ya que este texto se encuentra en el centro de todos los debates acerca del posfordismo y de la nueva cultura económica y social.

Boltanski y Chiapello ven en el posfordismo una nueva cultura empresarial que demanda a las empresas una menor rigidez organizativa para favorecer la «autocreación del trabajador». ${ }^{8}$ Es lo que ha dado en llamarse la teoría del capital humano, iniciada por Theodore Schultz (1961) y Gary Becker (1964), de gran importancia para la ideología neoliberal. El individuo, según ambos economistas, viene a ser su propio empresario, ya que debe invertir (su tiempo, su dinero, sus capacidades, etc.) en su propio desarrollo, que, si quiere tener éxito, debe estar enfocado a habilidades relacionadas con el mercado laboral. De este nuevo ethos surgen estructuras empresariales mucho más descentralizadas que las dominantes en el fordismo, donde el metarrelato subyacente implicaba una racionalización de casi todos los aspectos de la sociedad y, por consiguiente, también de la empresa, mucho más burocrática y autoritaria (Boltanski y Chiapello, 2002: 106-134).

Según Boltanski y Chiapello, este proceso flexibilizador implica una menor burocracia empresarial, lo cual conlleva una mayor flexibilidad individual a la hora de planificarse la vida laboral. Sin embargo, realmente esto no hace al empleado más libre, ya que su realización debe circunscribirse a los paráme-

7 Las empresas actuales no funcionan igual que las fordistas, tal como Boltanski y Chiapello (2002) y Alonso y Fernández (2018) nos han mostrado. La producción ya no está ni restringida a un número muy limitado de productos, ni es constante en el tiempo ni localizada en un complejo industrial. Los discursos gerenciales también han evolucionado para adaptarse a estas realidades. La alienación, en este sentido, también debe tomar nuevas y más dúctiles formas, ya no basta con el dominio material como en el fordismo.

8 Es importante señalar que las primeras indicaciones al respecto las hizo Foucault en $E l$ nacimiento de la biopolítica (2009: 306 y ss.), quien analizó las raíces y desarrollo del neoliberalismo, haciendo especial énfasis en las nuevas visiones del ser humano como homo oeconomicus, donde la teoría del capital humano cubre aspectos importantes relacionados con la educación y el mercado laboral. 
tros que marca el sistema. En definitiva, la inversión que se hace en esta autocreación debe ir encaminada a aumentar su productividad y flexibilidad. De este modo, esa creatividad, y esa autocreación que se le exige al empleado, es más bien un callejón sin salida donde los trabajadores se ven obligados a sacrificar su vida (y con ella su esencia más humana, la creatividad e imaginación) a cambio de un cierto estatus económico y social. En esto coincide Graeber con Boltanski y Chiapello. Sin embargo, nos gustaría apuntar algunas aportaciones de Graeber a estas intuiciones, casi dos décadas después de la publicación de El nuevo espíritu del capitalismo, y que bosquejan, a su juicio, el mercado laboral del capitalismo financiarizado actual.

En primer lugar, el antropólogo norteamericano considera que el sector dominante en la economía actual es el financiero, acerca de lo cual hay amplio consenso (Palazuelos, 2015: 303 y ss.), y por este motivo lo toma como ejemplo de funcionamiento del mercado laboral para otros sectores. Graeber habla del «feudalismo financiero»; veremos por qué y en qué sentido puede ser considerado como un ejemplo en la creación de empleo basura para el resto de sectores. En primer lugar, este sector no se dedica a la producción de nada real, simplemente a la puesta en circulación de dinero crediticio (Graeber, 2018: 167) y a moverlo frenéticamente, de tal modo que las empresas relacionadas con el sector puedan cobrar comisiones y penalizaciones por servicios relacionados con esas transacciones. Con el inicio del nuevo siglo, el arte de crear riqueza de este dinero inexistente e incontrolado acabó provocando un crecimiento desorbitado de la maquinaria del sistema financiero que desembocó en la Gran Recesión del 2008 (Stiglitz, 2010).

Este fue (y sigue siendo en gran medida), según Graeber, el caldo de cultivo perfecto para la generación de trabajos basura, ya que así se dota de apariencia de realidad al espejismo del submundo financiero. Muchos de los consejeros, equipos legales, actuarios, project managers y las cadenas interminables de ejecutivos intermedios sin una tarea reconocible son necesarios para justificar todo el sistema y para justificarse recíprocamente.

Como principio general, propondría lo siguiente: en todo sistema político-económico basado en la apropiación y la distribución de bienes (en vez de en su producción, asignación y mantenimiento), gran parte de su población se dedicará a redirigir esos recursos a lo largo y ancho del sistema, y tenderá a organizarse en complejas jerarquías con múltiples escalafones [...] Me gustaría añadir que la diferencia entre subordinados y siervos es a menudo demasiado confusa, puesto que la obediencia al superior suele ser una parte del trabajo mismo. La mayoría son señores a la par que vasallos (Graeber, 2018: 181). 
En el texto vemos que, en opinión de Graeber, el capitalismo financiarizado es un neofeudalismo por, en primer lugar, estar fuertemente estratificado, en este caso en dos grandes grupos sociales: ${ }^{2}$ los productores y los que viven del trabajo de los otros. En segundo lugar, por la fuerte jerarquización social, especialmente en los sectores más representativos, como es el sector financiero. En concreto, este segundo punto muestra que, después de todo, la flexibilización que los nuevos modos de cultura empresarial parecían augurar, tal como apuntaron Boltanski y Chiapello, han derivado, según Graeber, en justo lo contrario, en pirámides cuasi estamentales donde la obediencia es divisa y, consecuentemente, la creatividad solo puede ser expresada en los términos que el sistema dicta. Podría decirse que es el fin de una verdadera creatividad o, dicho de otro modo, el triunfo de la alienación. Por último, el antropólogo norteamericano señala como semejanza entre el feudalismo medieval y el financiero que los beneficios se realizan por apropiación, y no tanto por la producción y el comercio de excedentes.

En cualquier caso, hay algo en la posición de Graeber que no cuadra con la teoría económica más elemental: ¿qué empresa medianamente racional paga salarios que se podría ahorrar? Aquí es precisamente donde Bullshit Jobs entronca con En deuda. En ambos trabajos, en el fondo, se intenta demostrar que, tal como se da hoy en día, el capitalismo no tiene nada que ver con lo que la teoría entiende como tal. Si la teoría económica de la empresa privada afirma que el sentido de esta es proporcionar rendimiento económico a sus accionistas, los ejemplos que trae a colación Graeber en Bullshit Jobs dejan claro que al neofeudalismo financiero, al contrario de lo que ocurría en el fordismo industrial de posguerra, no le importan tanto los beneficios (si importasen, se eliminarían los empleos inútiles) y el mercado, cuanto la extracción de rentas. La lógica interna del sistema ha cambiado.

La existencia de los trabajos basura desafía la lógica del capitalismo. [...] Es un sistema de extracción de rentas donde las lógicas internas [...] tienen poco que ver con el capitalismo, puesto que los imperativos económicos y los políticos tienden a converger. En muchos aspectos recuerda al feudalismo medieval en el modo en que se despliegan innumerables jerarquías de señores, vasallos y siervos. [....] El hecho es que el presente sistema, en vez de haber reemplazado al capitalismo industrial, se le ha superpuesto, creando una realidad

9 Cuando hablamos de estratos, grupos sociales, etc. en sentido graeberiano de neofeudalismo, hay que tomarlos como aproximaciones o comparaciones, nunca al pie de la letra. El autor es consciente de las diferencias entre el feudalismo medieval y nuestra época actual, pero introduce esta noción con una intención más bien heurística. 
difícilmente discernible. La confusión es tan grande que ni tan siquiera los que se hallan sumidos en ella, pueden darle un sentido (Graeber, 2018: 191).

En este punto es donde cobra mayor fuerza su noción de neofeudalismo. La esencia del comportamiento feudal medieval, para Graeber, era la apropiación del botín robándolo directamente o creando tasas, diezmos o aduanas (Graeber, 2018: 176). En ese sentido afirma que lo económico y lo político están esencialmente entrelazados, es imposible discernirlos. Toda acción política, al tener una motivación económica (su fin era la apropiación de algo con valor económico), es también económica. Y viceversa: toda acción económica, al necesitar el apoyo del poder político, es a su vez política. Esa es también una de las principales características del feudalismo financiero del siglo XXI y que su teoría, en su opinión, saca a la luz y que explica, en gran medida, la causa de la proliferación desmesurada de los mismos.

El sistema extractivo del neofeudalismo actual se basa en la apropiación cuasi mafiosa de rentas, para la cual necesita una apariencia de realidad y prestigio. Por eso son necesarios, a juicio de Graeber, multitud de escalafones, cuadros de dirección y rangos departamentales. Es un sistema autónomo que se retroalimenta: necesita un tamaño considerable para poder aplicar la fuerza y arrebatar rentas, pero, cuanto más grande se vuelve, más empleados necesita, lo cual, a su vez, lo hace aumentar de tamaño y, por tanto, se necesitan más empleados. Ese es el proceso. Además, teniendo en cuenta que la esfera de lo económico ha engullido y anulado a la esfera de lo político, se desprende que la proliferación de todos esos trabajos es un fenómeno político y económico, a la vez que se explica en gran parte gracias a estas necesidades y al proceso de la financiarización.

\section{4. ¿POR QUÉ NADIE HACE NADA?}

En principio, cuando se plantean las críticas al capitalismo desde la perspectiva de la justicia, como, por ejemplo, puede ser entendida la crítica socialdemócrata, la respuesta a la pregunta «iPor qué nadie hace nada por cambiarlo?» es clara: porque hay muchos intereses creados. Es más, el capitalismo, desde esta óptica, podría considerarse como la puesta en juego de los distintos intereses y la competencia entre ellos. Esta postura nos lleva a considerar el capitalismo como esencialmente injusto. Sin embargo, si lo hacemos desde la perspectiva de Graeber, quien rechaza que el sistema actual sea de 
hecho un capitalismo, ya no podemos utilizar esa respuesta estándar de la competencia de los intereses. Así, para responder a la pregunta «iPor qué nadie hace nada?», el antropólogo estadounidense se centra en el «resentimiento moral».

Graeber, como venimos viendo, realiza una división entre los trabajadores que producen y los que no. Los no productivos son los que realizan lo que él denomina «trabajos basura» (más los desempleados, claro), mientras que los primeros llevan a cabo actividades productivas.

Si alguien hubiera diseñado un régimen laboral para mantener el poder del capital financiero, parece difícil imaginar que lo podría haber hecho mejor. Los trabajadores reales, los productivos, están siendo exprimidos y explotados sin compasión. El resto se dividen entre un estrato de aterrorizados desempleados y otro grupo, mucho más numeroso, que es pagado por no hacer nada, ocupando posiciones diseñadas para hacerles sentirse identificados con la clase dominante [...], pero que al mismo tiempo desarrollan un resentimiento contra cualquiera que realice un trabajo con una clara función social (Graeber, 2013b).

Ambos tipos de trabajadores (los que son productivos y los que no), influidos por la ideología neoliberal que sataniza a las clases más desfavorecidas, ${ }^{10}$ viven en el resentimiento hacia los excluidos del mercado laboral, al ser considerados como parásitos, gente que se aprovecha de los beneficios sociales estatales. A su vez, los desempleados se sienten abandonados e injustamente tratados y, por eso mismo, también experimentan animadversión frente a los que, al menos, pueden ganarse la vida con su propio trabajo. Por su parte, los individuos con empleos basura desarrollan un resentimiento sui géneris respecto a los trabajadores productivos (Graeber, 2018: 247).

En opinión de Graeber, la animadversión de los empleados de trabajos basura frente a los productivos se debe al fenómeno que él ha denominado «envidia moral» (Graeber, 2018: 248). El trabajador improductivo envidia a los que sí realizan un empleo socialmente productivo, no le importa tanto el sueldo o las comodidades que normalmente su tipo de trabajos suelen conllevar, sino que le duele que el suyo no tenga un sentido. Esto, qué duda cabe, está relacionado con la alienación de la que hablábamos anteriormente. Recordemos que, con este término, Graeber se refería a la pérdida de la «esencia humana», que era la capacidad de imaginar cosas y llevarlas a la práctica. Estar alienado, por consiguiente, implica perder la capacidad de imaginar, de producir y de ser reconocido socialmente por ello. La envidia moral es provocada

10 Véase, por ejemplo, Jones (2011). 
por esta alienación, con la particularidad de que el individuo que la sufre es consciente de ella (Graeber, 2018: 248).

Esta envidia moral también explicaría, afirma Graeber, fenómenos como la antipatía que grandes capas de la sociedad sienten respecto a los empleados públicos, y en especial hacia los docentes. Nadie con un cierto sentido social, en teoría, podría sentir algo parecido hacia ellos, dada la importante labor que desempeñan. Sin embargo, es por la importancia e impacto de su trabajo por lo que son envidiados. Otro ejemplo que propone Graeber como muestra de la veracidad de su tesis es el de la campaña intensiva de satanización de la clase obrera llevada a cabo por el neoliberalismo desde los gobiernos de Thatcher y Reagan (aunque, irónicamente, continuada por otros como los de Schröder y Blair). Sirvió para dinamitar el poder negociador de los sindicatos (Harvey, 2007), pero también para desprestigiar a toda la clase trabajadora. En efecto, siempre a juicio de Graeber, la clase empleada en los trabajos basura aceptará encantada las tesis neoliberales que califican a las clases trabajadoras como subsidiados sin escrúpulos y sin compromiso con su empresa, ya que así pueden justificar su envidia moral detrás de las teóricas miserias morales de los demás.

La realidad social que nos presenta Graeber parece cimentada en una especie de equilibrio de envidias entre los distintos tipos de empleados (y desempleados), que desemboca en una situación de equilibrio precario en la que nadie tiene ningún interés por cambiarla. Por ese motivo, nadie hace nada por cambiar la situación, porque, tal y como está diseñado el modelo socioeconómico actual, cualquier cambio puede resultar fatal para el mismo. Es un equilibrio imperfecto, donde todos los participantes, por miedo a empeorar todavía más, prefirieren conservar su estatus a arriesgar perderlo todo.

Después de todo, esta situación podría resumirse en lo que Graeber llama la «paradoja del trabajo contemporáneo», que consiste en que, a pesar de que la gente entiende que la dignidad personal y la autoestima están íntimamente relacionadas con el trabajo que realizamos para ganarnos la vida, la mayoría de la gente odia sus trabajos. Y esto, como ya hemos indicado, se debe a la alienación de gran parte de la sociedad, es decir, de los que sufren los bullshit jobs, individuos que se ven empujados a elegir entre encontrar un sentido a sus trabajos o a realizar trabajos realmente útiles, pero mal retribuidos y que a duras penas satisfacen las necesidades materiales (Graeber, 2018: 241 y ss.).

Esta situación, insiste Graeber, no es un problema económico como defiende la teoría económica dominante (las economics), sino más bien político. 
Nadie nos obliga a escoger nuestras profesiones, pero, de un modo u otro, la organización social en general y de los mercados laborales en particular, nos condicionan de tal modo que es casi imposible escapar del callejón sin salida donde nos encontramos. Los teóricos de la economía se han esforzado enormemente en reducir nuestro comportamiento a complejos modelos matemáticos que, pese a su sofisticación ecuacional, no consiguen dar cuenta de una manera sólida de nuestra naturaleza y de la creatividad que la identifica. A pesar de todo, la abstracción y el aura de misticismo que envuelve a la ciencia económica dotan de consistencia ideológica estas creencias y las convierten en algo más allá de lo político, algo científicamente irrefutable. El empeño, a nuestro juicio, de gran parte de la obra de Graeber consiste en desacralizar estas creencias y llevarlas al plano de lo político, en el cual, a priori, todos podemos decidir.

\section{CONCLUSIONES}

Pero, eso sí, para decidir entre todos, primero debemos rebatir las teorías en las que se cimenta la ideología neoliberal. Por un lado, la teoría de los trabajos basura puede ser entendida como una aproximación distinta a la explotación laboral, partiendo del concepto de alienación que Graeber ha desarrollado a lo largo de su obra y que nosotros hemos explicado en el primer apartado. Otro ámbito en el que la ideología neoliberal ha actuado con éxito como arma de dominación es el de la deuda como institución por la que se somete moralmente a individuos y pueblos (Graeber, 2014). Además de la burocracia, que para Graeber más que una ideología es un modo de hacer (aunque necesita un soporte teórico que la mantenga en pie), esos son los pilares del neoliberalismo financiarizado (Graeber, 2013a: 289 y ss.) y los que, por eso mismo, habría que socavar para poder aspirar a un sistema político de corte asambleario, autónomo y solidario, que es al que él, como anarquista, aspira (2009, 2013a).

En el caso concreto de los trabajos basura, sorprende en principio que preste tanta atención a los problemas de los trabajadores del sistema financiero y, sin embargo, casi no se mencionen los trabajadores de cuello azul. Puede ser que, efectivamente, en los países más desarrollados la proporción de trabajadores con este tipo de trabajos sea elevada, pero ¿es así a escala mundial? Graeber presenta escasos datos comparativos entre sectores, circunscritos a algunos países en concreto, pero a escala mundial, por mucho que hayan au- 
mentado en términos absolutos los trabajos basura, ¿podemos afirmar que el verdadero problema de la explotación y la alienación de los trabajadores es tal y como él la define? Es difícil creer que el problema global del capitalismo sea que muchos trabajadores de los centros financieros mundiales no encuentren un sentido a su trabajo. Es posible, y de hecho consideramos su propuesta muy imaginativa, que este fenómeno arroje luz sobre muchos aspectos de las sociedades de algunos países occidentales, pero no creemos que baste para explicar la problemática de la explotación a gran escala.

En cualquier caso, creemos que el gran aporte de Graeber con este libro consiste en detectar un nuevo fenómeno que, puesto en perspectiva con los estudios actuales de sociólogos como Green, Dejours, Boltanski o Moruno puede dar una idea más completa de la naturaleza de la alienación y de los modos materiales y simbólicos empleados por las élites. Por ejemplo, Dejours (2015) se asemeja a Graeber en la importancia que da al «sufrimiento en el trabajo» por la falta de reconocimiento. Sennet, por su lado, nos muestra la problemática de la ruina personal (del carácter) que supone una vida laboral sin expectativas humanamente enriquecedoras. Green profundiza en la demanda agotadora y poco reconfortante de grandes grupos de trabajadores en el capitalismo avanzado. La crisis del trabajo, por consiguiente, ya no es de carácter estrictamente material, sino (sobre todo) moral.

Con independencia de las grandes diferencias que hay entre ellos, lo realmente relevante es el interés por la vertiente más humana de los estudios críticos con el capitalismo. En efecto, una vez superadas la época del economicismo marxista y la del individualismo abstracto de la teoría económica, el foco de atención de los que cuestionan el capitalismo se centra en los desafíos que se nos plantean en el sistema actual, que tienen que ver con el desprecio a lo humano que supone la falta de objetivos en la vida, el estrés en el trabajo, el nulo reconocimiento o, en el caso de Graeber, la anulación de la capacidad creativa del ser humano.

Zygmunt Bauman entendió a la perfección el giro humanista de la crítica social al analizar, por ejemplo, los «residuos humanos» que la modernidad, por su propia inercia, iba produciendo (Bauman, 2013). El progreso, según el pensador polaco, genera residuos, y estos no son solo ecológicos o económicos, sino, sobre todo, humanos. Ningún diseño, como lo es el del crecimiento económico del capitalismo actual, puede incluir todos los casos, todas las realidades que componen el mundo globalizado. Tal y como indicó Karl Polanyi en La gran transformación, todo el entramado del sistema económico y social del capitalismo moderno, que toma su forma actual en la desintegración del orden 
mundial del siglo XIX, se basa en el interés individual, con lo cual, lo que se entiende como progreso estará en gran medida relacionado con las exigencias de los intereses privados, y no con los del conjunto de la sociedad.

Ese es en gran medida el punto de vista de Graeber (2001), quien cree que debemos reformular los modos en que valoramos, ya que solo así será posible imaginarse una sociedad en cuya construcción podamos participar políticamente todos. La economía debe depender del tejido social, y no a la inversa. Por eso, la obra de Graeber se centra en el estudio y crítica de los soportes de la sociedad actual, como un paso previo a la revolución. Ahí reside la vocación crítica y alternativa de Graeber. Es consciente, por un lado, de la importancia de la acción directa como ejemplo de lo que puede ser un sistema político conformado por individuos libres y autónomos (Graeber, 2002, 2009 y 2011, especialmente el del 2009). Pero no por ello descuida la crítica teórica de la ideología neoliberal (Graeber, 2014, 2015 y 2018).

Desde la perspectiva del giro (revolucionario) que va de una sociedad dominada por el interés individual a una en la que, tal y como defiende Graeber, los individuos autónomos se asocian libremente, es como debemos entender, en nuestra opinión, la obra de Graeber y, en especial, su Bullshit Jobs. Diríamos que su antropología moral debe ser estudiada como una teoría de y para la práctica $\mathrm{o}$, dicho de otro modo, de y para la revolución emancipadora del ser humano. Creemos que, desde este punto de vista, se esté de acuerdo o no con él, merece especial atención, por la originalidad de sus propuestas, por los diálogos que abre con otras corrientes de la teoría radical y de otras disciplinas $\mathrm{y}$, además, por ser una de las escasas voces anarquistas de la academia.

\section{BIBLIOGRAFÍA}

Alonso, Luis Enrique y Fernández, Carlos J. (2018). Poder y sacrificio. Los nuevos discursos de la empresa. Madrid: Siglo XXI.

Bauman, Zygmunt (2013). Vidas desperdiciadas: La modernidad y sus parias. Barcelona: Paidós.

Becker, Gary (1964). Human Capital: A Theoretical and Empirical Analysis. Chicago: The University of Chicago Press.

Boltanski, Luc y Chiapello, Eve (2002). El nuevo espíritu del capitalismo. Madrid: Akal.

Debord, Guy (2005). La sociedad del espectáculo. Valencia: Pretextos. 
Dejours, Christophe (2015). El sufrimiento en el trabajo. Buenos Aires: Topía.

Foucault, Michel (2008). Nacimiento de la biopolítica. Curso en el Collége de France (1978-1979), Buenos Aires: Fondo de cultura Económica.

Graeber, David (2001). Toward an Anthropological Theory of Value. New York: Palgrave.

Graeber, David (2002). The New Anarchist. New Left Review, 13, 61-74.

Graeber, David (2009). Direct Action. An Ethnography. Edinburgh: AK Press.

Graeber, David (2011). Fragmentos de una antropología anarquista. Barcelona: Virus Editorial.

Graeber, David (2013a). The Democracy Project. A History, A Crisis, A Movement. New York: Spiegel \& Grau.

Graeber, David (2013b). On the Phenomenon of the Bullshit Jobs: A work Rant. Strike, August 2013.

Graeber, David (2014). Debt. The First 5000 years. Brooklyn: Melville House.

Graeber, David (2015). La utopía de las normas. De la tecnología, la estupidez y los secretos placeres de la burocracia. Barcelona: Ariel.

Graeber, David (2018). Bullshit Jobs. A Theory. London: Allan Lane/Penguin Books.

Green, Francis (2004). Work Intensification, Discretion, and the Decline in Well Being at Work. Eastern Economic Journal, 30(4): 615-625.

Green, Francis (2007). Demanding Work: The Paradox of Job Quality in the Affluent Economy. Princeton: Princeton University Press. Green, Francis, Felstead, Alan y Gallie, Duncan (2015). Unequal Britain at Work. Oxford: Oxford University Press.

Harvey, David (2007). Breve historia del neoliberalismo. Madrid: Akal. Jones, Owen (2011). Chavs: The Demonization of the Working Class. London / New York: Verso. 
Marx, Karl (1997). Manuscritos de economía y filosofía. Madrid: Alianza Editorial.

Moruno, Jorge (2018). No tengo tiempo. Geografías de la precariedad. Madrid: Akal.

Palazuelos, Enrique (Dir., 2015). Economía política mundial. Madrid: Akal.

Polanyi, Karl (2017). La gran transformación. Ciudad de México: FCE.

Schultz, Theodore W. (1961). Investment in human capital. American Economic Review, 51(1), 1-17.

Sennett, Richard (2000). La corrosión del carácter: las consecuencias personales del trabajo en el nuevo capitalismo. Barcelona: Anagrama.

Sennett, Richard (2006). La cultura del nuevo capitalismo. Barcelona: Anagrama.

Vaneigem, Raoul (1967). The Revolution of Everyday Life. London: Rebel Press.

Srnicek, Nick y Williams, Alex (2017). Inventar el futuro. Poscapitalismo y un mundo sin trabajo. Barcelona/México D. F.: Malpaso.

Standing, Guy (2013). El precariado. Una nueva clase social. Barcelona: Pasado y Presente.

Standing, Guy (2017a). La corrupción del capitalismo: Por qué proliferan los rentistas y trabajar no sale a cuenta. Barcelona: Pasado y Presente.

Standing, Guy (2017b). El advenimiento del precariado. Entrevista. Sin Permiso. Recuperado de: http://www.sinpermiso.info/textos/eladvenimiento-del-precariado-entrevista [Consultado el 26 de noviembre de 2018].

Stiglitz, Joseph (2010). Freefall. America, Free Markets, and the Sinking of the World Economy. New York/London: W. W. Norton \& Company. 